\title{
Metformin Ameliorates Lipopolysaccharide-Induced Depressive-Like Behaviors and Abnormal Glutamatergic Transmission
}

Jiang Chen

Nanchang University

Tian Zhou

Nanchang University

Wen-Bin Chen

Nanchang University

Dong Lin

Nanchang University

A-Min Guo

Nanchang University

Zi-Yang Liu

Nanchang University

Erkang Fei ( $\nabla$ fek@ncu.edu.cn )

Nanchang University https://orcid.org/0000-0002-3581-9521

Research

Keywords: Depression, Metformin, Lipopolysaccharide (LPS), Synaptic transmission

Posted Date: July 28th, 2020

DOl: https://doi.org/10.21203/rs.3.rs-48554/v1

License: (c) (i) This work is licensed under a Creative Commons Attribution 4.0 International License. Read Full License

Version of Record: A version of this preprint was published at Biology on October 26th, 2020. See the published version at https://doi.org/10.3390/biology9110359. 


\section{Abstract}

\section{Background}

Metformin, a first-line drug for type 2 diabetes mellitus (T2DM), has been found to reduce depressive symptoms in patients comorbid depression with other diseases. However, it is largely unclear that how metformin ameliorates the depressive-like behaviors.

\section{Methods}

Lipopolysaccharide (LPS) was injected intraperitoneally into C57BL/6 mice to induce depressive-like behaviors, and metformin was administrated in LPS-induced depression mouse model. Forced swimming test (FST) and tail suspension test (TST) were employed to detect the depressive-like behaviors. Wholecell patch clamp recording in the hippocampal pyramidal neurons was adopted to record the miniature excitatory postsynaptic currents (mEPSCs) and paired-pulse ratios (PPR).

\section{Results}

We found LPS-treated mice exhibited increased immobility in FST and TST, and elevated glutamatergic transmission. Furthermore, metformin administration in the LPS-treated mice ameliorated depressive-like behaviors and abnormal glutamatergic transmission.

\section{Conclusion}

Our results suggest that metformin have antidepressant effects and can correct abnormal glutamatergic transmission, providing an insight to the underlying mechanism of metformin on depression.

\section{Background}

Depression is a common and disabling mental illness with an estimated lifetime prevalence of 1 in 6 people [1], and has been identified as one of the leading contributors to the global health burden [2]. Depression is characterized by a persistent low mood, loss of interest in normally enjoyable activities, reduced self-esteem, insomnia, and suicide [3]. Current antidepressant medications or treatments are associated with limited symptomatic efficacy and delayed response [4]. Meanwhile, depression is interrelated to diabetes. The risk for depression is double in type 2 diabetes mellitus (T2DM) patients [5, 6], which imply depression and T2DM may share biological pathways, and treatments for T2DM may also alleviate depression.

The biguanide metformin is the first-line drug for T2DM therapy with a long history. Metformin lowers the plasma glucose level and enhances insulin sensitivity [7]. Intriguingly, it is also found that metformin may reduce depressive symptoms in patients comorbid depression with other disorders [8, 9]. A case study [8] reported that metformin and spironolactone (a mineralocorticoid receptor antagonist) treatments relieved the depression in a young woman with depression and Polycystic Ovarian Syndrome (PCOS). In another 
clinical study [9], 58 patients diagnosed with depression and T2DM were divided into two groups for metformin or placebo treatment for 24 weeks, and it was found that metformin improved depressive symptoms. And recently, metformin was also found to be effective as an adjunct to antidepressants in nondiabetic major depressive disorder (MDD) patients [10]. Moreover, metformin is also found to ameliorate depressive-like behaviors in rodent models [11-13]. In a rat T2DM model induced by streptozotocin and nicotinamide, foot-shocks were administrated to model comorbid depression in diabetic rats. And metformin decreased the immobility time during the forced swimming test (FST) in this rat model of comorbid depression and diabetic [11]. In mice fed with high fat diet (HFD), metformin decreased the immobility time in the tail suspension test (TST) [12]. In mice subjected to chronic social defeat stress (CSDS), metformin treatment rescued the depressive-like behaviors in social interaction behavioral test, sucrose preference test, FST and TST [13]. Metformin also alleviates the depressive-like behaviors in sucrose preference test and FST in chronic unpredictable mild stress (CUMS) mice model [14]. These studies suggested metformin may exert anti-depressant effects. However, it is largely unclear that how metformin ameliorates the depressive-like behaviors.

Glutamate is the major excitatory neurotransmitter that is released at synapses in the brain. Dysfunction of glutamatergic neurotransmission is implicated in the pathogenesis of depression [15-17]. It has been reported that glutamate level is increased in the serum, cerebrospinal fluid and brain tissues of patients with depression [18-20]. And altered expression or function of glutamate N-methyl-d-aspartate receptors (NMDARs) subunits is also found in depression patients [21-25]. In rodent stress models of depression, acute stress increases glutamate release, while chronic stress leads to neuronal atrophy in the prefrontal cortex and hippocampus, and alters glutamatergic transmission [26-29].

In this study, we used lipopolysaccharide (LPS) to induce depressive-like behaviors in mice, and found that glutamatergic transmission was increased in LPS induced depression mouse model. Next, we examined whether metformin ameliorates depressive-like behaviors and rescues elevated glutamatergic transmission in LPS induced depression mouse model.

\section{Materials And Methods}

\section{Animals and drug treatments}

Aged 6 7 weeks male C57BL/6 mice, which were purchased from model animal research center of Nanjing University (Nanjing, China), were used in the experiments. All mice were housed in a constant temperature and humidity chamber at $25^{\circ} \mathrm{C}$, and sufficient food and water were administered daily. No more than 5 adult mice per cage were subjected to a 12-hour light/dark cycle under standard conditions. All the mice were guaranteed to be hygienic. The animal experiments were carried out in accordance with the "Guidelines for the Care and Use of Laboratory Animals" promulgated by Nanchang University.

LPS (Sigma, L-2880) was dissolved in sterile, endotoxin-free $0.9 \%(\mathrm{~m} / \mathrm{v})$ sodium chloride $(\mathrm{NaCl})$ solution (saline) and was injected intraperitoneally (i.p.) into mice at a dosage of $0.5 \mathrm{mg} / \mathrm{kg}$ (bodyweight) to stimulate subclinical infection without causing significant inflammation and other significant damage to 
the animal [30]. After 10-day (once daily) administration of LPS, the mice were subjected to behavioral tests and electrophysiological or other experiments.

Metformin (Sigma) was dissolved in $0.9 \%(\mathrm{~m} / \mathrm{v}) \mathrm{NaCl}$ solution (vehicle) and was i.p. injected into mice at a dosage of $200 \mathrm{mg} / \mathrm{kg}$ (bodyweight) based on previous studies [31]. After 10-day LPS treatment and following behavioral tests, the mice were administrated daily with Metformin or its vehicle for another 10 days before another behavioral tests and electrophysiological or other experiments.

\section{Behavioral tests}

For forced swim test (FST), mice were individually placed in a 2-liter (L) beaker containing clean water $\left(23-25^{\circ} \mathrm{C}\right)$. The depth of water is $20 \mathrm{~cm}$ to prevent the animal from touching the bottom with the tail or hind legs. Mice behaviors were videotaped from the side for 6 min under red light. The immobility time of the last 4 minutes was recorded by an observer blinded to animal treatments. Immobility was defined as the time that animals remained floating or stationary.

For tail suspension test (TST), mice were suspended individually about $50 \mathrm{~cm}$ above the floor by fixing the tail with a medical tape on the test box hook. Mice behaviors were videotaped from the side for 6 min under red light. The last 4 minutes of rest time was recorded by an observer blinded to animal treatment. Immobility was defined as the time when animals stopped struggling.

\section{Electrophysiological recording}

Preparation of hippocampal slices as described previously [32]. The mice were anesthetized with ketamine/xylazine (Sigma, 100/20 mg/kg, respectively, ip), brain was quickly removed in ice-cold partial sucrose solution (PSS) containing (in $\mathrm{mM}$ ): ( $80 \mathrm{NaCl}, 3.5 \mathrm{KCl}, 4.5 \mathrm{MgSO}_{4}, 0.5 \mathrm{CaCl}_{2}, 1.25 \mathrm{NaH}_{2} \mathrm{PO}_{4}, 25$ $\mathrm{NaHCO}_{3}, 10 \mathrm{D}-(+)$-Glucose, 90 Sucrose). Coronal hippocampal slices $(300 \mu \mathrm{m})$ were cut in ice-cold PSS using a VT-1000S vibratome (Leica, Germany). Then transferred hippocampal slices into traditional artificial cerebrospinal fluid (ACSF) (in mM): (124 NaCl, $2.5 \mathrm{KCl}, 2 \mathrm{MgSO} 4,2.5 \mathrm{CaCl} 2,1.25 \mathrm{NaH} 2 \mathrm{PO} 4,26$ NaHCO3, 10 D-(+)-Glucose) were incubated for $30 \mathrm{~min}$ at $34{ }^{\circ} \mathrm{C}$, and 1 hour at room temperature (25 \pm 1 ${ }^{\circ} \mathrm{C}$ ) before recording. All solutions contained saturated 95\% 02 / 5\% CO2 (vol / vol).

Slices were transferred into a recording chamber perfused with a $2-3 \mathrm{ml} / \mathrm{min}$ ACSF at $32-34{ }^{\circ} \mathrm{C}$. CA1 pyramidal neurons were observed using an upright microscope equipped with an infrared sensitive CCD camera (C2400-75, Hamamatsu) and a 40 'soaking lens (Axioskop 2 Plus, Zeiss). Borosilicate pipette were filled with an intracellular solution with a resistance of 3-5 $\mathrm{M} \Omega$. Whole-cell patch clamp recording were made using the Multiclamp 700B amplifier, Digidata 1440A interface digitization, pClamp 10.5 software acquisition at $2 \mathrm{kHz}$.

For mEPSC recording, adding $1 \mu \mathrm{M}$ TTX and $20 \mu \mathrm{M}$ bicuculline to ACSF to block action potential and inhibitory synaptic transmission, respectively. The pipettes were filled with potassium gluconate intracellular solution containing (in mM): $130 \mathrm{~K}$-gluconate, $5 \mathrm{NaCl}_{1} 1 \mathrm{MgCl}_{2}, 10 \mathrm{HEPES}, 0.2 \mathrm{EGTA}, 2 \mathrm{Mg}$ - 
ATP, 0.1 Na-GTP, 5 QX-314 (pH 7.30, 293 m0sm). The hippocampal CA1 pyramidal neurons were held at $-70 \mathrm{mV}$ recorded by whole-cell patch clamp method in the voltage-clamp mode.

For electrical properties of hippocampal CA1 pyramidal neurons detect, adding $20 \mu \mathrm{M}$ bicuculline $50 \mu \mathrm{M}$ DL-AP5 and20 $\mu \mathrm{M}$ DNQX to ACSF blocked excitatory and inhibitory synaptic transmission. The pipette solution containing (in mM): 130 K-gluconate, $5 \mathrm{NaCl}, 1 \mathrm{MgCl}_{2}, 10$ HEPES, $0.2 \mathrm{EGTA}, 2 \mathrm{Mg}$-ATP, $0.1 \mathrm{Na}$ GTP (pH 7.30, 294 mOsm). The whole-cell patch clamp recording method was used to record in the current clamp mode, and action potential was recorded by injecting depolarizing pulses (from 0 to 350 $\mathrm{pA})$ at a step of $50 \mathrm{pA}$ per $10 \mathrm{~s}$.

For paired-pulse ratios recording, adding $20 \mu \mathrm{M}$ bicuculline and $5 \mu \mathrm{M}$ CGP52432 blocked inhibitory synaptic transmission. The Pipettes were filled with potassium gluconate intracellular solution containing (in mM): 130 K-gluconate, $5 \mathrm{NaCl}, 1 \mathrm{MgCl} 2,10$ HEPES, 0.2 EGTA, $2 \mathrm{Mg}$-ATP, $0.1 \mathrm{Na}-\mathrm{GTP}, 5 \mathrm{QX}-314$ (pH 7.30, $293 \mathrm{mOsm}$ ). EPSCs were stimulated by a paired stimulus of the same size at intervals of 100, 50, and $25 \mathrm{~ms}$ in SC-CA1 pathway. The ratio value is calculated as [(p2 / p1) × 100], where $\mathrm{p} 2$ and $\mathrm{p} 1$ are the amplitudes of the EPSCs induced by the second pulse and the first pulse, respectively.

\section{Brain morphological analysis}

Mice were anesthetized by intraperitoneal injection of $0.7 \%$ sodium pentobarbital. After $5-10$ min of perfusion of saline, the mice were perfused with pre-cold paraformaldehyde for 5-10 min, brain was weighed and fixed in $4 \%$ PFA at $4{ }^{\circ} \mathrm{C}$ overnight. Slices $(50 \mu \mathrm{m})$ were cut in ice-cold PBS using a VT-1000S vibratome (Leica, Germany). After washing with $0.01 \mathrm{M}$ PBS, Sections were attached to a gelatin-coated slide and air-dried at room temperature. Samples were incubated in a cresol purple solution, heated by water bath in $60^{\circ} \mathrm{C}$ for 3-10 min. Then washing with Milli-Q water for 3 times, samples were put into $75 \%$, $95 \%$ and $100 \%$ ethanol for 1 min respectively. Samples were immersed in xylene for 10 min, sealed with a neutral resin overnight, and photographed with a fluorescence microscope (FN1هNikon).

\section{Statistical analysis}

The data were statistically analyzed and graphed using the Graphpad Prism 6.0 software. Electrophysiological data were analyzed using Clampfit and Mini-Analysis software. Images were edited using Photoshop CS3. The experimental data were expressed as Min to Max or Mean \pm SEM. Student's ttests were used to compare the two groups of data. One-way ANOVA was used to compare three or more groups of data. Two-way ANOVA was used in electrophysiological studies when more than two parameters were analyzed. ${ }^{*} p<0.05 ; * \star p<0.01 ; * \star \star p<0.001$.

\section{Results}

\section{LPS induces depressive-like behaviors in mice}


LPS has been reported to produce depressive-like behaviors, including increased immobility in forced swimming test (FST) and tail suspension test (TST), in rodents [33,34]. To phenotype depressive-like behaviors in C57BL/ 6 mice, we injected intraperitoneally (i.p.) $0.5 \mathrm{mg} / \mathrm{kg}$ LPS into wild type (WT) male mice daily for 10 days (Fig. 1a). Meanwhile, mice injected with the vehicle (saline) were served as the control. Compared with the control, LPS treatment didn't alter mice brain size, weight, as well as the structure of cortex and hippocampus (Fig. S1). After 10 day-treatment, the mice were subjected to FST and TST sequentially in the next 2 days to confirm the depressive-like behaviors. As shown in Fig. 1b-e, mice treated with LPS exhibited more immobility time than control mice in both FST (Fig. 1b and c) and TST (Fig. 1d and e). These results indicate LPS induces depressive-like behaviors in our mouse model successfully.

\section{Miniature excitatory postsynaptic currents (mEPSCs) frequency of hippocampal pyramidal neurons is increased in LPS induced depression mouse model}

To further detect whether LPS treatment affect synaptic transmission, we recorded mEPSCs in hippocampal CA1 pyramidal neurons. Recording was performed in the presence of tetrodotoxin (TTX) and bicuculine to block action potentials and $y$-aminobutyric acid (GABA) transmission, respectively. As shown in Fig. 2a and c, no difference was observed in mEPSC amplitude between LPS treated mice and control. However, mEPSC frequency was increased in mice treated with LPS (Fig. 2a and b), suggesting LPS treatment increases glutamatergic transmission.

\section{Metformin Ameliorates Lps-induced Depressive-like Behaviors}

To detect whether metformin can ameliorate LPS-induced depressive-like behaviors, we administrated LPS-induced depression mice model with metformin. As shown in Fig. 3a, after i.p. injection of LPS, or saline, for 10 days and behavioral tests (FST and TST), the mice were then i.p. injected with $200 \mathrm{mg} / \mathrm{kg}$ metformin, or its vehicle, daily for another 10 days. Totally, we divided these mice into four groups: I. Saline + Vehicle; II. Saline + Metformin; III. LPS + Vehicle; IV. LPS + Metformin. Brain size, weight, as well as the structure of cortex and hippocampus were observed, and no changes were found between the mice of these four groups (Fig. S2). We also evaluated the depressive-like behaviors in these four groups with FST and TST. Like the results in Fig. 1, LPS alone treatment (group III vs. I) increased the immobility level in FST and TST (Fig. 3b and c). However, administration of metformin in LPS-treated mice (group IV vs. III) decreased the immobility level to that of group I (Fig. 3b and c). These results suggest that metformin ameliorates LPS-induced depressive-like behaviors.

\section{Metformin reduces increased mEPSCs frequency in LPS induced depression mouse model}

mEPSC frequency was increased in LPS-induced depression mice, and metformin ameliorated LPSinduced depressive-like behaviors, we asked if metformin can reduce increased mEPSC frequency in LPStreated mice. mEPSCs were recorded in hippocampal CA1 pyramidal neurons from these four groups. 
Similar as the results in Fig. 2, LPS alone treatment (group III vs. I) increased mEPSC frequency (Fig. 4a and b). And administration of metformin in LPS-treated mice (group IV vs. III) reduced the mEPSC frequency to that of group I (Fig. $4 a$ and b). mEPSC amplitudes were not altered between these four groups. These results suggest that metformin rescues the increased mEPSC frequency in LPS induced depression mice.

\section{Metformin reduces presynaptic glutamate release in LPS induced depression mouse model}

To determine if LPS and metformin change the intrinsic excitability of pyramidal neurons in hippocampus, we observed the neuronal firing response upon different depolarizing currents injection in the current clamp mode. As shown in Fig. S3, there wasn't any change in number of action potentials (APs) between the four different groups, suggesting neither LPS nor metformin affects the excitability of hippocampal CA1 pyramidal neurons.

Next, we wonder if the changed mEPSC frequencies in Figs. 2 and 4 were attributed to the altered presynaptic glutamate release. We measured EPSCs evoked by two presynaptic stimulations delivered at different intervals (i.e., paired pulses), and the fraction of EPSC2/EPSC1 amplitudes was defined as the paired-pulse ratios (PPR). As shown in Fig. 5, PPR at $25 \mathrm{~ms}$ and $50 \mathrm{~ms}$ intervals were decreased in LPS alone treated mice (group III vs. I), but administration of metformin in LPS-treated mice (group IV vs. III) increased the PPR to that of group I. These results suggest glutamate release probability is increased in LPS-induced depression mice, and metformin rescues the increased glutamate release.

\section{Discussion}

Here we demonstrate that metformin ameliorates depressive-like behaviors and glutamatergic transmission in LPS induced depression mouse model. First, the mice administrated with LPS showed increased immobility in FST and TST, which represent the depressive-like behaviors, and increased mEPSC frequency in the hippocampal pyramidal neurons. Second, the increased immobility in FST and TST, as well as increased mEPSC frequency in LPS-treated mice were reduced after the administration of metformin. Third, neither LPS nor metformin treatment didn't alter the excitability of hippocampal neurons. However, presynaptic glutamate release, which was measured by PPR, in LPS-induced depression mice was increased, but was decreased after the administration of metformin. These results present evidence that metformin ameliorates depressive-like behaviors and glutamatergic neurotransmission in LPS induced depression mouse model.

The bacterial endotoxin LPS is a characteristic component of the outer membrane of Gram-negative bacteria and is a cytokine inducer to activate acutely the peripheral innate immune system.

Administration of LPS in rodent models induces depressive-like behaviors, like increased immobility in the FST and TST, decreased consumption of saccharin solutions, suppression of sexual behavior, and attenuation of cocaine-induced place preference [33-35]. And some depressive-like behaviors can also be attenuated by chronic antidepressant treatment [33]. So LPS-treated mice have been used as a depression model for novel antidepressants screening $[36,37]$. Here, we also observed that LPS-treated 
mice exhibited increased immobility time in the FST and TST (Fig. 1). We used the LPS-treated mice as a depression model to evaluate if metformin ameliorates depressive-like behaviors in this model.

It has been demonstrated that LPS treatment also have roles in synaptic transmission and plasticity. Exposure of rat cortical slices to LPS induces a rapid and sustained release of glutamate [38]. LPS suppresses rat hippocampal long-term potential (LTP) in vitro [39, 40] and in vivo [41]. And paired-pulse facilitation (PPF) or PPR is reduced in LPS-treated rats, which suggest LPS increases presynaptic release probability [41]. In vitro acute exposure of brain slices to LPS enhances excitatory synaptic transmission and neuronal excitability [42]. Here, we indeed observed LPS-treated mice exhibited increased mEPSC frequency and reduced PPR (Figs. 2, 4 and 5), suggesting increased presynaptic glutamate release. But our in vivo data showed that neuronal excitability wasn't changed in LPS-treated mice (Fig. S3). Taken together, these data indicate glutamatergic transmission is elevated in LPS-treated mice.

Although metformin is the mainstay of therapy for T2DM with a long history, it has been also indicated that metformin may have effects in some neurological disorders, like neurodevelopmental disorders, neurodegenerative diseases, and neuropsychiatric disorders. In a fragile $X$ syndrome (FXS) mice model, i.p. injection of metformin corrects core deficits, like social deficit, repetitive behavior, abnormal dendritic spine morphology and exaggerated long term depression (LTD) [43]. And there are language, cognitive and behavioral improvements in a small sample size of metformin treated FXS patients [44-46]. In an Alzheimer's disease (AD) mice model, metformin attenuates amyloid plaque deposition and spatial memory deficit [47]. Metformin also have neuroprotective effects for dopaminergic neurons in 1-methyl-4phenyl-1,2,3,6-tetrahydropyridine (MPTP)-induced Parkinson's disease (PD) mice model [48, 49]. Moreover, metformin is found to rescue the early brain changes and abnormal behaviors in a Huntington's disease (HD) mice model [50].

For depression, metformin can reduce depressive symptoms in T2DM patients with depression [9]. In mice, metformin ameliorates depressive-like behaviors induced by CSDS [13] or CUMS [14]. A recent study reported that administration of metformin at $100 \mathrm{mg} / \mathrm{kg}$ and $200 \mathrm{mg} / \mathrm{kg}$, but not $300 \mathrm{mg} / \mathrm{kg}$, rescued the increased immobility in FST and TST in LPS-treated male Swiss albino mice [51]. Consistent with this result, we also found that the increased immobility time in FST and TST were reduced after administration of metformin at $200 \mathrm{mg} / \mathrm{kg}$ in LPS-treated male C57BL/6 mice (Fig. 3). Not only depressive-like behaviors, but also elevated glutamatergic transmission was rescued upon metformin administration in LPS-treated mice. In our study, we found LPS-treated mice exhibited increased mEPSC frequency and reduced PPR, and metformin ameliorated these deficits (Figs. 2, 4 and 5). LPS is a cytokines (like interleukin-6 (IL-6) and tumor necrosis factor-a (TNF-a)) inducer to activate immune system, and metformin has anti-inflammation effects and can suppress the induction of IL- 6 and TNF-a by LPS [52]. Many cytokines are also found in the brain and regulate neuronal excitability, synaptic transmission and plasticity. The rescue of glutamatergic transmission deficits by metformin in LPStreated mice may be attribute to the anti-inflammation effect of metformin, which need our further examination. 


\section{Conclusions}

Taken together, our study suggested that metformin ameliorates depressive-like behaviors induced by LPS, and the anti-depressant effect of metformin may be carried out through its downregulation of glutamatergic transmission in LPS-induced depression mouse model. Here we provided an insight to the underlying mechanism of metformin on depression.

\section{Abbreviations}

LPS lipopolysaccharide

T2DM type 2 diabetes mellitus

FST forced swimming test

TST tail suspension test

PCOS polycystic ovarian syndrome

MDD major depressive disorder

HFD high fat diet

CUMS chronic unpredictable mild stress

NMDARs N-methyl-d-aspartate receptors

i.p. intraperitoneally

ACSF artificial cerebrospinal fluid

mEPSCs miniature excitatory postsynaptic currents

GABA y-aminobutyric acid

TTX tetrodotoxin

APs action potentials

PPR paired-pulse ratios

PPF paired-pulse facilitation

LTP long-term potential

LTD long-term depression 
MPTP 1-methyl-4-phenyl-1,2,3,6-tetrahydropyridine

FXS fragile $X$ syndrome

AD Alzheimer's disease

PD Parkinson's disease

HD Huntington's disease

CSDS chronic social defeat stress

IL-6 interleukin-6

TNF-a tumor necrosis factor-a

\section{Declarations}

Ethics approval and consent to participate

This study was approved by the Research Ethics Committee of Nanchang University (Nanchang, China).

Consent for publication

All the co-authors consent to publish the work in Cell \& Bioscience.

Availability of data and materials

All data generated or analyzed during this study are included in this published article (and its supplementary information files).

Competing interests

The authors declare that there is no conflict of interest.

\section{Funding}

This work was supported by grants from National Natural Science Foundation of China ( 81460215 , 31771142, 31860268).

\section{Authors' contributions}

Conceived and designed the experiments: JC, TZ and EF. Performed the experiments: JC, TZ and AG. Analyzed the data: JC, TZ, WC, DL, ZL and EF. Wrote the paper: JC, TZ and EF. All authors read and approved the final manuscript. 
Not applicable.

\section{References}

1. Kessler RC, Berglund P, Demler O, Jin R, Merikangas KR, Walters EE. Lifetime prevalence and age-ofonset distributions of DSM-IV disorders in the National Comorbidity Survey Replication. Arch Gen Psychiatry. 2005;62(6):593-602. doi:10.1001/archpsyc.62.6.593.

2. Whiteford HA, Degenhardt L, Rehm J, Baxter AJ, Ferrari AJ, Erskine HE, et al. Global burden of disease attributable to mental and substance use disorders: findings from the Global Burden of Disease Study 2010. Lancet. 2013;382(9904):1575-86. doi:10.1016/S0140-6736(13)61611-6.

3. Belmaker RH, Agam G. Major depressive disorder. N Engl J Med. 2008;358(1):55-68. doi:10.1056/NEJMra073096.

4. Gaynes BN, Warden D, Trivedi MH, Wisniewski SR, Fava M, Rush AJ. What did STAR*D teach us? Results from a large-scale, practical, clinical trial for patients with depression. Psychiatr Serv. 2009;60(11):1439-45. doi:10.1176/ps.2009.60.11.1439.

5. Ali S, Stone MA, Peters JL, Davies MJ, Khunti K. The prevalence of co-morbid depression in adults with Type 2 diabetes: a systematic review and meta-analysis. Diabet Med. 2006;23(11):1165-73. doi:10.1111/j.1464-5491.2006.01943.x.

6. Anderson RJ, Freedland KE, Clouse RE, Lustman PJ. The prevalence of comorbid depression in adults with diabetes: a meta-analysis. Diabetes Care. 2001;24(6):1069-78. doi:10.2337/diacare.24.6.1069.

7. Pernicova I, Korbonits M. Metformin-mode of action and clinical implications for diabetes and cancer. Nat Rev Endocrinol. 2014;10(3):143-56. doi:10.1038/nrendo.2013.256.

8. Rasgon NL, Carter MS, Elman S, Bauer M, Love M, Korenman SG. Common treatment of polycystic ovarian syndrome and major depressive disorder: case report and review. Curr Drug Targets Immune Endocr Metabol Disord. 2002;2(1):97-102.

9. Guo M, Mi J, Jiang QM, Xu JM, Tang YY, Tian G, et al. Metformin may produce antidepressant effects through improvement of cognitive function among depressed patients with diabetes mellitus. Clin Exp Pharmacol Physiol. 2014;41(9):650-6. doi:10.1111/1440-1681.12265.

10. Abdallah MS, Mosalam EM, Zidan AA, Elattar KS, Zaki SA, Ramadan AN, et al. The Antidiabetic Metformin as an Adjunct to Antidepressants in Patients with Major Depressive Disorder: A Proof-ofConcept, Randomized, Double-Blind, Placebo-Controlled Trial. Neurotherapeutics. 2020. doi:10.1007/s13311-020-00878-7.

11. Shivavedi N, Kumar M, Tej G, Nayak PK. Metformin and ascorbic acid combination therapy ameliorates type 2 diabetes mellitus and comorbid depression in rats. Brain Res. 2017;1674:1-9. doi:10.1016/j.brainres.2017.08.019.

12. Zemdegs J, Martin H, Pintana H, Bullich S, Manta S, Marques MA, et al. Metformin Promotes Anxiolytic and Antidepressant-Like Responses in Insulin-Resistant Mice by Decreasing Circulating 
Branched-Chain Amino Acids. J Neurosci. 2019;39(30):5935-48. doi:10.1523/JNEUROSCI.290418.2019.

13. Fang W, Zhang J, Hong L, Huang W, Dai X, Ye Q, et al. Metformin ameliorates stress-induced depression-like behaviors via enhancing the expression of BDNF by activating AMPK/CREB-mediated histone acetylation. J Affect Disord. 2020;260:302-13. doi:10.1016/j.jad.2019.09.013.

14. Liu W, Liu J, Huang Z, Cui Z, Li L, Liu W, et al. Possible role of GLP-1 in antidepressant effects of metformin and exercise in CUMS mice. J Affect Disord. 2019;246:486-97. doi:10.1016/j.jad.2018.12.112.

15. Duman RS, Sanacora G, Krystal JH. Altered Connectivity in Depression: GABA and Glutamate Neurotransmitter Deficits and Reversal by Novel Treatments. Neuron. 2019;102(1):75-90. doi:10.1016/j.neuron.2019.03.013.

16. Duman RS, Aghajanian GK, Sanacora G, Krystal JH. Synaptic plasticity and depression: new insights from stress and rapid-acting antidepressants. Nat Med. 2016;22(3):238-49. doi:10.1038/nm.4050.

17. Murrough JW, Abdallah CG, Mathew SJ. Targeting glutamate signalling in depression: progress and prospects. Nat Rev Drug Discov. 2017;16(7):472-86. doi:10.1038/nrd.2017.16.

18. Kim JS, Schmid-Burgk W, Claus D, Kornhuber HH. Increased serum glutamate in depressed patients. Arch Psychiatr Nervenkr (1970). 1982;232(4):299-304.

19. Levine J, Panchalingam K, Rapoport A, Gershon S, McClure RJ, Pettegrew JW. Increased cerebrospinal fluid glutamine levels in depressed patients. Biol Psychiatry. 2000;47(7):586-93. doi:10.1016/s0006-3223(99)00284-x.

20. Hashimoto K, Sawa A, lyo M. Increased levels of glutamate in brains from patients with mood disorders. Biol Psychiatry. 2007;62(11):1310-6. doi:10.1016/j.biopsych.2007.03.017.

21. Karolewicz B, Stockmeier CA, Ordway GA. Elevated levels of the NR2C subunit of the NMDA receptor in the locus coeruleus in depression. Neuropsychopharmacology. 2005;30(8):1557-67. doi:10.1038/sj.npp.1300781.

22. Karolewicz B, Szebeni K, Gilmore T, Maciag D, Stockmeier CA, Ordway GA. Elevated levels of NR2A and PSD-95 in the lateral amygdala in depression. Int J Neuropsychopharmacol. 2009;12(2):143-53. doi:10.1017/S1461145708008985.

23. Feyissa AM, Chandran A, Stockmeier CA, Karolewicz B. Reduced levels of NR2A and NR2B subunits of NMDA receptor and PSD-95 in the prefrontal cortex in major depression. Prog Neuropsychopharmacol Biol Psychiatry. 2009;33(1):70-5. doi:10.1016/j.pnpbp.2008.10.005.

24. Chandley MJ, Szebeni A, Szebeni K, Crawford JD, Stockmeier CA, Turecki G, et al. Elevated gene expression of glutamate receptors in noradrenergic neurons from the locus coeruleus in major depression. Int J Neuropsychopharmacol. 2014;17(10):1569-78. doi:10.1017/S1461145714000662.

25. Gray AL, Hyde TM, Deep-Soboslay A, Kleinman JE, Sodhi MS. Sex differences in glutamate receptor gene expression in major depression and suicide. Mol Psychiatry. 2015;20(9):1057-68. doi:10.1038/mp.2015.91. 
26. Lowy MT, Gault L, Yamamoto BK. Adrenalectomy attenuates stress-induced elevations in extracellular glutamate concentrations in the hippocampus. J Neurochem. 1993;61(5):1957-60. doi:10.1111/j.1471-4159.1993.tb09839.x.

27. Musazzi L, Milanese M, Farisello P, Zappettini S, Tardito D, Barbiero VS, et al. Acute stress increases depolarization-evoked glutamate release in the rat prefrontal/frontal cortex: the dampening action of antidepressants. PLoS One. 2010;5(1):e8566. doi:10.1371/journal.pone.0008566.

28. Li N, Liu RJ, Dwyer JM, Banasr M, Lee B, Son H, et al. Glutamate N-methyl-D-aspartate receptor antagonists rapidly reverse behavioral and synaptic deficits caused by chronic stress exposure. Biol Psychiatry. 2011;69(8):754-61. doi:10.1016/j.biopsych.2010.12.015.

29. $10.1038 / \mathrm{nrn} 3138$

increases As, rat d-egrit, of pfctda, antidepressantsPopoli M, Yan Z, McEwen BS, et al. The stressed synapse: the impact of stress and glucocorticoids on glutamate transmission. Nat Rev Neurosci. 2011;13(1):22-37. doi: 10.1038/nrn3138.

30. Sulakhiya K, Keshavlal GP, Bezbaruah BB, Dwivedi S, Gurjar SS, Munde N, et al. Lipopolysaccharide induced anxiety- and depressive-like behaviour in mice are prevented by chronic pre-treatment of esculetin. Neurosci Lett. 2016;611:106-11. doi:10.1016/j.neulet.2015.11.031.

31. Wang J, Gallagher D, DeVito LM, Cancino GI, Tsui D, He L, et al. Metformin activates an atypical PKCCBP pathway to promote neurogenesis and enhance spatial memory formation. Cell Stem Cell. 2012;11(1):23-35. doi:10.1016/j.stem.2012.03.016.

32. Chen YJ, Zhang M, Yin DM, Wen L, Ting A, Wang P, et al. ErbB4 in parvalbumin-positive interneurons is critical for neuregulin 1 regulation of long-term potentiation. Proc Natl Acad Sci U S A. 2010;107(50):21818-23. doi:10.1073/pnas.1010669107.

33. Yirmiya R. Endotoxin produces a depressive-like episode in rats. Brain Res. 1996;711(1-2):163-74. doi:10.1016/0006-8993(95)01415-2.

34. Frenois F, Moreau M, O'Connor J, Lawson M, Micon C, Lestage J, et al. Lipopolysaccharide induces delayed FosB/DeltaFosB immunostaining within the mouse extended amygdala, hippocampus and hypothalamus, that parallel the expression of depressive-like behavior. Psychoneuroendocrinology. 2007;32(5):516-31. doi:10.1016/j.psyneuen.2007.03.005.

35. Suzuki T, Funada M, Sugano Y, Misawa M, Okutomi T, Soma G, et al. Effects of a lipopolysaccharide from Pantoea agglomerans on the cocaine-induced place preference. Life Sci. 1994;54(6):PL75-80. doi:10.1016/0024-3205(94)00704-7.

36. Custodio CS, Mello BS, Cordeiro RC, de Araujo FY, Chaves JH, Vasconcelos SM, et al. Time course of the effects of lipopolysaccharide on prepulse inhibition and brain nitrite content in mice. Eur $\mathrm{J}$ Pharmacol. 2013;713(1-3):31-8. doi:10.1016/j.ejphar.2013.04.040.

37. Tomaz VS, Cordeiro RC, Costa AM, de Lucena DF, Nobre Junior HV, de Sousa FC, et al. Antidepressant-like effect of nitric oxide synthase inhibitors and sildenafil against lipopolysaccharide-induced depressive-like behavior in mice. Neuroscience. 2014;268:236-46. doi:10.1016/j.neuroscience.2014.03.025. 
38. Wang YS, White TD. The bacterial endotoxin lipopolysaccharide causes rapid inappropriate excitation in rat cortex. J Neurochem. 1999;72(2):652-60. doi:10.1046/j.1471-4159.1999.0720652.x.

39. Jo JH, Park EJ, Lee JK, Jung MW, Lee CJ. Lipopolysaccharide inhibits induction of long-term potentiation and depression in the rat hippocampal CA1 area. Eur J Pharmacol. 2001;422(1-3):6976. doi:10.1016/s0014-2999(01)01075-5.

40. Cunningham AJ, Murray CA, O'Neill LA, Lynch MA, O'Connor JJ. Interleukin-1 beta (IL-1 beta) and tumour necrosis factor (TNF) inhibit long-term potentiation in the rat dentate gyrus in vitro. Neurosci Lett. 1996;203(1):17-20. doi:10.1016/0304-3940(95)12252-4.

41. Commins S, O'Neill LA, O'Mara SM. The effects of the bacterial endotoxin lipopolysaccharide on synaptic transmission and plasticity in the CA1-subiculum pathway in vivo. Neuroscience. 2001;102(2):273-80. doi:10.1016/s0306-4522(00)00498-x.

42. Gao F, Liu Z, Ren W, Jiang W. Acute lipopolysaccharide exposure facilitates epileptiform activity via enhanced excitatory synaptic transmission and neuronal excitability in vitro. Neuropsychiatr Dis Treat. 2014;10:1489-95. doi:10.2147/NDT.S65695.

43. Gantois I, Khoutorsky A, Popic J, Aguilar-Valles A, Freemantle E, Cao R, et al. Metformin ameliorates core deficits in a mouse model of fragile X syndrome. Nat Med. 2017;23(6):674-7. doi:10.1038/nm.4335.

44. Dy ABC, Tassone F, Eldeeb M, Salcedo-Arellano MJ, Tartaglia N, Hagerman R. Metformin as targeted treatment in fragile X syndrome. Clin Genet. 2018;93(2):216-22. doi:10.1111/cge.13039.

45. Protic D, Aydin EY, Tassone F, Tan MM, Hagerman RJ, Schneider A. Cognitive and behavioral improvement in adults with fragile $X$ syndrome treated with metformin-two cases. Mol Genet Genomic Med. 2019;7(7):e00745. doi:10.1002/mgg3.745.

46. Biag HMB, Potter LA, Wilkins V, Afzal S, Rosvall A, Salcedo-Arellano MJ, et al. Metformin treatment in young children with fragile X syndrome. Mol Genet Genomic Med. 2019;7(11):e956. doi:10.1002/mgg3.956.

47. Ou Z, Kong X, Sun X, He X, Zhang L, Gong Z, et al. Metformin treatment prevents amyloid plaque deposition and memory impairment in APP/PS1 mice. Brain Behav Immun. 2018;69:351-63. doi:10.1016/j.bbi.2017.12.009.

48. Patil SP, Jain PD, Ghumatkar PJ, Tambe R, Sathaye S. Neuroprotective effect of metformin in MPTPinduced Parkinson's disease in mice. Neuroscience. 2014;277:747-54. doi:10.1016/j.neuroscience.2014.07.046.

49. Lu M, Su C, Qiao C, Bian Y, Ding J, Hu G. Metformin Prevents Dopaminergic Neuron Death in MPTP/P-Induced Mouse Model of Parkinson's Disease via Autophagy and Mitochondrial ROS Clearance. Int J Neuropsychopharmacol. 2016;19(9). doi:10.1093/ijnp/pyw047.

50. Arnoux I, Willam M, Griesche N, Krummeich J, Watari H, Offermann N, et al. Metformin reverses early cortical network dysfunction and behavior changes in Huntington's disease. Elife. 2018;7. doi:10.7554/eLife.38744. 
51. Mudgal J, Nampoothiri M, Basu Mallik S, Kinra M, Hall S, Grant G, et al. Possible involvement of metformin in downregulation of neuroinflammation and associated behavioural changes in mice. Inflammopharmacology. 2019;27(5):941-8. doi:10.1007/s10787-019-00638-w.

52. Kim J, Kwak HJ, Cha JY, Jeong YS, Rhee SD, Kim KR, et al. Metformin suppresses lipopolysaccharide (LPS)-induced inflammatory response in murine macrophages via activating transcription factor-3 (ATF-3) induction. J Biol Chem. 2014;289(33):23246-55. doi:10.1074/jbc.M114.577908.

\section{Figures}

Fig. 1

a

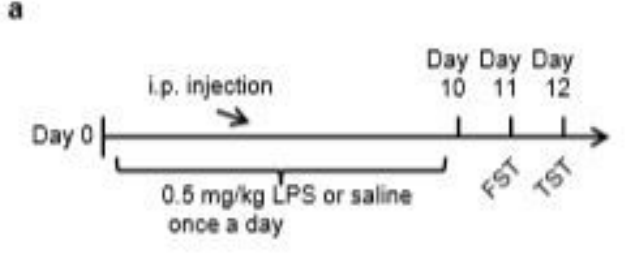

d

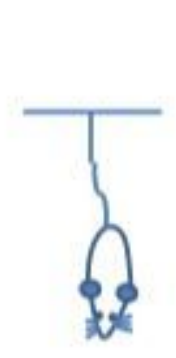

e b

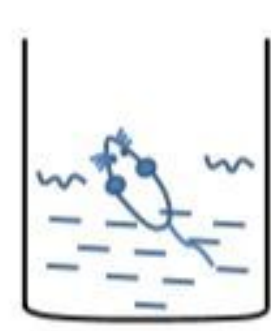

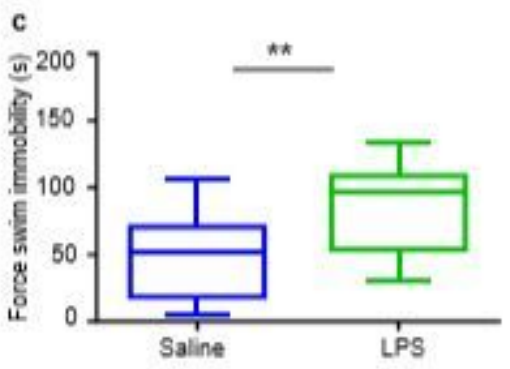

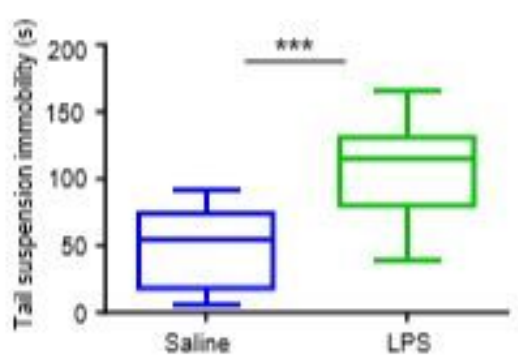

\section{Figure 1}

LPS induces depressive-like behaviors in mice. a Schematic illustration of experimental procedure for LPS administration and behavioral tests for depressive-like behaviors in mice. C57BL/ 6 male mice at age of $6 \sim 7$ weeks were intraperitoneally (i.p.) injected with $0.5 \mathrm{mg} / \mathrm{kg}$ LPS or equal volume of saline daily for 10 days. The forced swimming test (FST) and tail suspension test (TST) were conducted at 24 hours (hrs) and $48 \mathrm{hrs}$ after the LPS treatment. After the behavioral tests, these mice were sacrificed for brain morphological analysis. b and c LPS treatment significantly increased the immobility time in FST. b Schematic diagram of mouse FST. c Quantification of the immobility time (seconds, s) in 4 minutes (mins) for each group mice in FST. Data were shown as mean \pm SEM ( $n=14$ mice for each group, Student's t-test, $\left.{ }^{* *} p<0.01\right)$. $d$ and e LPS treatment significantly increased the immobility time in TST. $d$ Schematic diagram of mouse TST. e Quantification of the immobility time (s) for each group mice in TST. Data were shown as mean \pm SEM $(n=14$ mice for each group, Student's t-test, $* \star * p<0.001)$. 
a

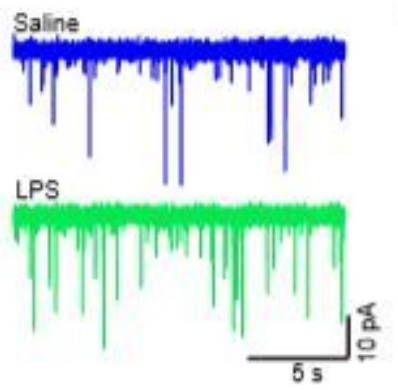

b

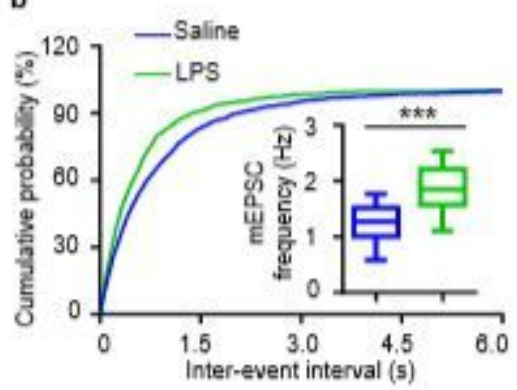

c

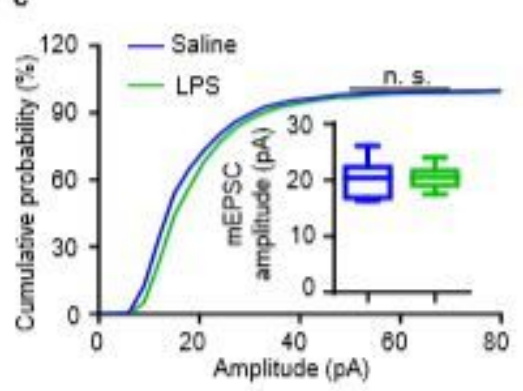

Figure 2

mEPSCs frequency of hippocampal pyramidal neurons is increased in LPS induced depression mouse model. a Representative traces of mEPSCs in CA1 pyramidal neurons from saline-treated (upper panel, blue) or LPS-treated (lower panel, green) mice. $b$ and c Cumulative probability plots of mEPSC inter-event intervals and histograms of mEPSC frequency (b) and amplitude (c) $(n=14$ neurons from 3 saline-treated mice; $\mathrm{n}=16$ neurons from 3 LPS-treated mice, Student's t-test, $* \star * p<0.001$, n.s. $p>0.05$ ).

Fig. 3
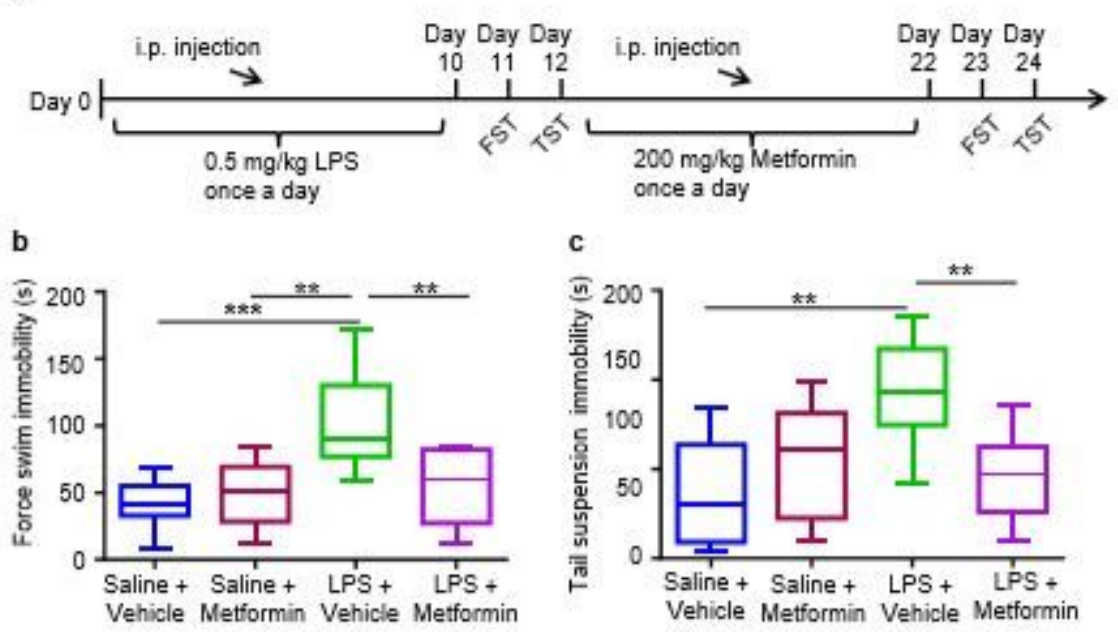

\section{Figure 3}

Metformin ameliorates LPS-induced depressive-like behaviors. a Schematic illustration of experimental procedure for LPS and metformin administration, and behavioral tests for depressive-like behaviors in mice. C57BL/ 6 male mice at age of $6 \sim 7$ weeks were i.p. injected with $0.5 \mathrm{mg} / \mathrm{kg}$ LPS or equal volume of saline daily for 10 days. After selection through TST and FST, the mice exhibited depressive-like behaviors were i.p. injected with $200 \mathrm{mg} / \mathrm{kg}$ metformin or equal volume of its vehicle for another 10 days. Then FST and TST were conducted at $24 \mathrm{hrs}$ and $48 \mathrm{hrs}$ after the metformin treatment. $\mathrm{b}$ and c LPS inducing increased immobility time in FST and TST was reduced by metformin treatment. Quantification of the immobility time (s) in 4 mins for each group mice in FST (b) and TST (c) ( $n=8 \sim 9$ mice for each group, one-way ANOVA, $* * p<0.005, * \star * p<0.001$ ). 

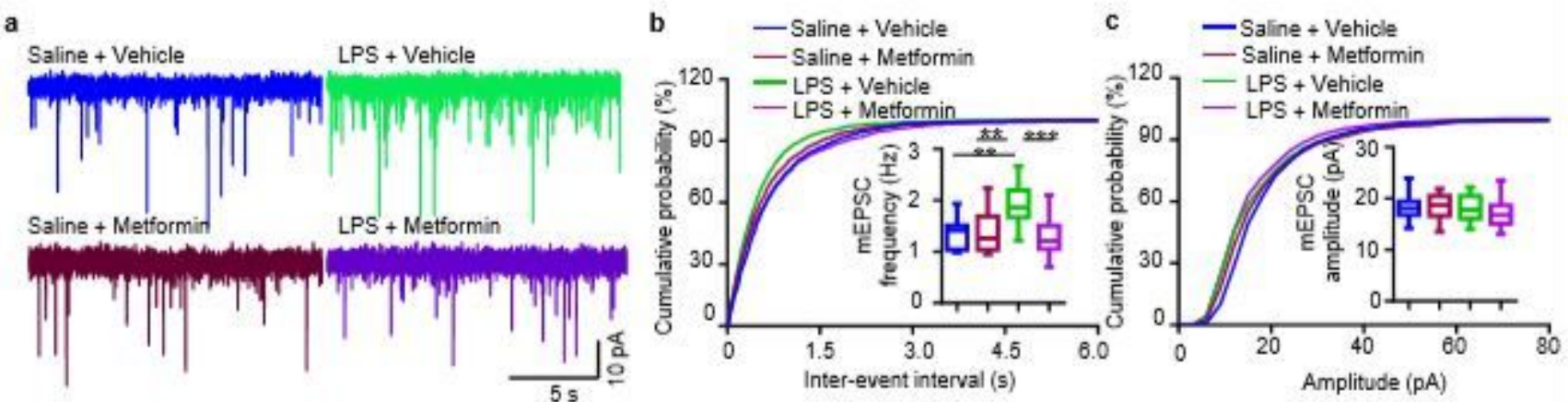

\section{Figure 4}

Metformin reduces increased mEPSCs frequency in LPS induced depression mouse model. a

Representative traces of mEPSCs in CA1 pyramidal neurons from mice of Saline + Vehicle (blue), Saline + Metformin (brown), LPS + Vehicle (green) and LPS + Metformin (purple) four different groups. b and c Cumulative probability plots of mEPSC inter-event intervals and histograms of mEPSC frequency (b) and amplitude (c) ( $n=14 \sim 16$ neurons from 3 mice for each group; one-way ANOVA, $* \star p<0.005, * \star * p<$ $0.001)$.

Fig. 5
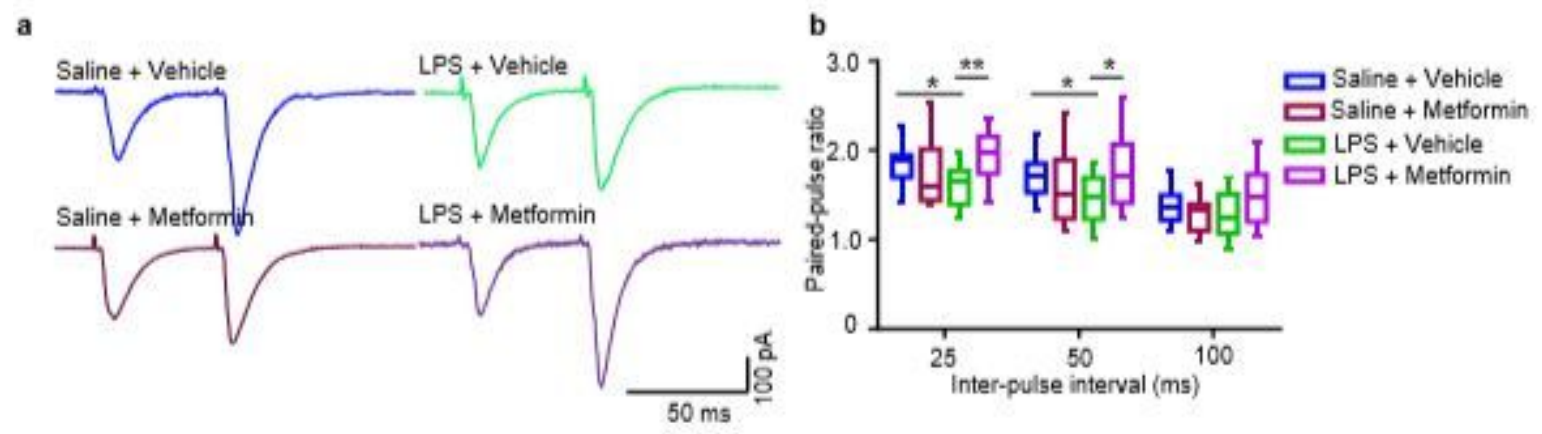

Figure 5

Metformin reduces presynaptic glutamate release in LPS induced depression mouse model. a Representative traces of PPR in CA1 pyramidal neurons from mice of Saline + Vehicle (blue), Saline + Metformin (brown), LPS + Vehicle (green) and LPS + Metformin (purple) four different groups. b PPRs plotted against inter-stimulus intervals ( $n=13 \sim 14$ neurons from 3 mice for each group; two-way ANOVA, $* p<0.05, * * p<0.005)$.

\section{Supplementary Files}

This is a list of supplementary files associated with this preprint. Click to download.

- Additionalfile1.pdf 